\title{
Bregs in Chronic HBV: Is It Time for Bragging Rights?
}

\author{
Nadia Alatrakchi
}

Published online: 17 February 2015

(c) Springer Science+Business Media New York 2015

Hepatitis B virus (HBV) infection is a major global health concern and is the most common cause of chronic liver disease worldwide. Despite the availability of a prophylactic vaccine, HBV infects around 400 million people worldwide, with endemic areas in Asia and Africa.

HBV is an extremely infectious virus that is spread when infected blood, semen, or another body fluid enters the body of non-infected person and can also be spread from mother to infant at birth. Infection with HBV can be either acute or chronic; while adult infections have a relatively low rate of chronicity (around $5 \%$ ), neonatal infections usually have a high persistence rate. Chronic infection is usually asymptomatic for decades (except in rare fulminant), but HBV carriers are at risk of developing life-threatening cirrhosis and eventually hepatocellular carcinoma (HCC). Currently, more than 780,000 people die every year due to the (rare) acute or (more common) chronic consequences of hepatitis B. It is estimated that more than 240 million people have chronic (long-term) HBV liver infection (1.2 million in US). For many of these individuals, the current forms of therapy lack efficacy mainly because pathogenic mechanisms responsible for acute and chronic hepatitis B remain only partly clarified.

\section{Effector T Cell Response in HBV Infection}

The immune system is a major influence on the course and outcome of hepatitis B [1]. In patients with self-limited acute hepatitis B, both effector CD4+ T helper and CD8+

N. Alatrakchi $(\bowtie)$

Massachusetts General Hospital, Harvard Medical School, GI Unit/Warren 10, 55 Fruit St., Boston, MA 02114, USA e-mail: nalatrakchi@mgh.harvard.edu cytotoxic $\mathrm{T}$ cell (CTL) responses to viral antigens are strong and multispecific, whereas these responses are weak or undetectable in chronically infected patients. The effector immune system also appears to be important for maintaining recovery, as strong and broad antiviral $\mathrm{T}$ cell responses are usually maintained for decades after clinical recovery. Patients with either inactive or resolved hepatitis $\mathrm{B}$ can experience reactivation with return of high levels of HBV antigens and DNA upon immunosuppression, emphasizing the importance of virus-specific immunity in maintaining control of viral replication. Importantly, patients with persistent $\mathrm{HBV}$ infection harbor weak immune response to $\mathrm{HBV}$. Understanding how $\mathrm{HBV}$-specific $\mathrm{T}$ cell responses become blunted during chronic HBV infection is essential to develop efficient therapies.

\section{Regulatory B Cell Response in HBV Infection}

Immune-suppressor cells such as $\mathrm{CD} 4^{+} \mathrm{CD} 25^{\text {high }}$ FoxP $^{+}$ regulatory $\mathrm{T}$ (Treg) cells exert multiple, negative regulatory functions on the effector immune cells, contributing substantially to the impairment of HBV clearance. Recently, a new family of regulatory cells, regulatory B cells (Bregs), was reported to control immune responses at the innate and the adaptive levels [2], and only few studies, including the present paper, have investigated the role of Breg cells in chronic hepatitis B (CHB) [3, 4].

B lymphocytes were initially identified for their production of cell surface antibody and their subsequent differentiation into plasma cells and consequent antibody secretion, with their antigen-presenting function later identified. Emerging significant evidence indicates that B cells can also actively modulate immune responses through mechanisms that do not directly involve the production of 
antibodies [2]. These B cells are functionally defined "regulatory B cells," or Breg, possibly acting earlier in the immune response than Treg cells [5]. Expansion of Bregs inhibits harmful immune responses to chronic inflammation by suppressing effector $\mathrm{T}$ cells, either through contactdependent or independent mechanisms [6]. The best understood mechanism by which Bregs suppress immune function is through secretion of IL-10, which inhibits the production of pro-inflammatory cytokines and supports regulatory $\mathrm{T}$ cell differentiation. The immune regulatory mechanisms of Bregs defined to date are protection from lethal inflammation, alteration of the development of autoimmune diseases, inhibition of anticancer responses in tumor models and possibly contribution to allograft tolerance [7]. Nevertheless, little is known of the contribution of Bregs to immune function in human persistent viral infections such as in chronic viral hepatitis.

Currently, few studies have investigated the influence of Breg cells in chronic hepatitis B. A pioneering study performed by Das et al. [3] reported increased Breg cells in the peripheral blood of patients with $\mathrm{CHB}$ who underwent spontaneous hepatic flare, demonstrating suppressive IL-10 mediated activity of Breg on $\mathrm{HBV}$-specific CD8 cells in vitro. Interestingly, Breg temporally associated with the spontaneous flare, reflected by increased viral load and liver inflammation. This could suggest that Breg are responsible for the flare by suppressing HBV-specific CD8 cells and therefore diminishing viral control. On the other hand, it is possible that HBV flare induced a CD8-specific $\mathrm{T}$ cell response, which was subsequently followed by an increase in Breg response to protect against the exaggerated immune activation. Most probably in the setting of chronic infection, both scenarios are happening and further studies are needed to understand the balance.

In this issue of the DDS [4], Gong et al. further confirmed accumulation of Breg in patients with chronic HBV infection. The authors studied Breg interaction with CD4+ $\mathrm{T}$ cell subsets (i.e., Th1 and Treg), reporting a modest but statistically significant inverse correlation between circulating IL-10 producing Bregs and T helper 1 (Th1) cells, suggesting that Bregs downregulated Th1 cells. Both studies suggest that Breg cells regulate $\mathrm{T}$ cell immunity in CHB. How such interactions affect the disease outcome remain to be further addressed.

Breg contribution to infection-related immunity is also described in HIV and schistosomiasis infections. Breg producing IL-10 may limit schistosomiasis-related liver disease [8]. In human immunodeficiency virus (HIV)-1 infection, IL-10-producing B cells, which suppress HIV-1specific $\mathrm{T}$ cell responses in vitro, are elevated in early and chronic HIV-1 infection [9]. Such expansion of Breg may suppress the generalized immune activation that characterizes HIV infection. Bregs also contribute to HIV-1 infection-associated immune dysfunction by impairing $\mathrm{T}$ cell function via IL-10 secretion and possibly programmed death ligand 1 (PD-L1) expression [10].

\section{Regulatory B Cells Phenotype and Function}

In their work, Gong et al. simply define Breg cells as $\mathrm{CD} 19+\mathrm{IL}-10^{+}$, a fair characterization given the current knowledge of the Breg phenotype. In fact, several phenotypes have been reported to define Bregs in mice and humans, raising the possibility that distinct subsets of Breg exist in different tissues and compartments in vivo [7, 11].

Murine Bregs predominantly exhibit a CD19+CD $1 d^{\text {high }}$ CD5+ phenotype in the spleen. They may also present as $\mathrm{CD} 19+\mathrm{CD} 21^{\text {high }} \mathrm{IgM}^{\text {high }}$ splenic $\mathrm{B}$ cells, either with or without expression of $\mathrm{CD} 23$, and exert their immunosuppressive function in an IL-10 dependent manner. In humans, IL-10 producing B cells are present in the peripheral blood, detected in the $\mathrm{CD} 19+\mathrm{CD} 27-\mathrm{CD} 24^{\text {high }} \mathrm{CD} 38^{\text {high }}$ subset of B cells. After CD40 stimulation, these cells suppress the differentiation of Th1 cells, partially via the provision of IL-10. Their suppressive capacity is reversed via blockade with CD80 and CD86 monoclonal antibodies, suggesting a contact-dependent suppressive action. Others described IL-10 producing $\mathrm{B}$ cells within the memory $\mathrm{CD} 24^{\text {high }} \mathrm{CD} 27+\mathrm{B}$ cell population. These Breg cells were able to negatively regulate monocyte cytokine production through IL-10-dependent pathways. Recently, a connectivity map analysis revealed that IL-10+ B cells are those specifically undergoing differentiation toward a germinal center B cell fate [11]. These cells were identified as CD11c-negative B cells, had suppressive activity, and were unable to efficiently differentiate into plasma cells.

Taken together, characterization of Bregs is still developing, with general surface markers for Bregs still not well defined and efficient IL-10 production their only consistent distinguishing feature. While Bregs are usually recognized as CD19+IL-10+, Breg secreting TGF- $\beta$ have also been described [12].

\section{Breg Versus Treg}

Interestingly, Gong et al. also report a positive correlation between the increased abundance of Bregs and Tregs in the circulation in chronic HBV patients. Similarities and differences between these two types of regulatory cells are here briefly overviewed.

Similar to Tregs, Bregs exert their suppressive properties through several mechanisms: Th1 and Th17 differentiation inhibition, Treg induction, and also through a direct inhibitory effect on antigen presentation by dendritic cells 
[3, 4, 13-15]. Breg subtypes may be homologous to Treg subtypes ("Br1" cells expressing IL-10, "Br3" cells expressing TGF- $\beta$ ), although the $\mathrm{Br} 1$ subtype seems to predominate. There also seems to be a number of suppression modalities, such as through secretion of IL-10 and TGF- $\beta$, and through the expression of pro-apoptotic membrane molecules that vary across Breg subtypes. Nevertheless, differences with Treg cells exist: Breg activation may chiefly involve the Toll-like receptors rather than the antigen receptor; Bregs may act earlier, facilitating the recruitment of Tregs, then disappearing once the Tregs become operational. In the tumor microenvironment, Bregs induce conversion from resting CD4 $\mathrm{T}$ cells to Tregs to support metastatic growth and promote Tregs in HCC [1315]. Breg increase FoxP3 and CTLA-4 expression in Treg cells, which was mainly dependent on direct contact between Breg and Treg cells, but was also TGF- $\beta$ although not IL-10 dependent [16].

Since B cells and $\mathrm{T}$ cells have the potential to infiltrate the liver $[3,17]$, they are possible therapeutic targets in the immunological control of liver inflammation. In the setting of chronic persistent hepatitis B virus infection, Breg and Treg are upregulated, suppressing persistent inefficient effector $\mathrm{T}$ cell response in the liver. It will be interesting to further explore how interactions between Tregs and Bregs contribute to the persistence of viral infections, as combining strategies targeting regulatory cell types could be beneficial.

\section{Summary}

Understanding of the interactions of viral hepatitis pathogens with the immune system is essential in the quest to slow the progression of liver disease in virus-infected subjects. It is to be hoped that gaining increased knowledge of the contribution of Breg in HBV-related immunity will enable the identification of novel targets for immunotherapy.

\section{References}

1. Alatrakchi N, Koziel MJ. Antiviral T-cell responses and therapy in chronic hepatitis B. J Hepatol. 2003;39:631-634.
2. Mauri C, Ehrenstein MR. The 'short' history of regulatory B cells. Trends Immunol. 2008;29:34-40.

3. Das A, Ellis G, Pallant C, et al. IL-10-producing regulatory B cells in the pathogenesis of chronic hepatitis B virus infection. $J$ Immunol. 2012;189:3925-3935.

4. Gong Y, Zhao C, Zhao P, Wang M, Zhou G, Han F, Cui Y, Qian J, Zhang H, Xiong H, Sheng J, Jiang T. Role of IL-10-producing regulatory B cells in chronic hepatitis B virus infection. Dig Dis Sci. (Epub ahead of print). doi:10.1007/S10620-014-3358-1.

5. Berthelot JM, Jamin C, Amrouche K, Le Goff B, Maugars Y, Youinou P. Regulatory B cells play a key role in immune system balance. Joint Bone Spine. 2013;80:18-22.

6. Vitale G, Mion F. Regulatory B cells: Evidence, developmental origin and population diversity. Mol Immunol. 2010;48:1-8.

7. Chesneau M, Michel L, Degauque N, Brouard S. Regulatory B cells and tolerance in transplantation: from animal models to human. Front Immunol. 2013;4:497.

8. van der Vlugt LE, Labuda LA, Ozir-Fazalalikhan A, et al. Schistosomes induce regulatory features in human and mouse CD1d(hi) B cells: inhibition of allergic inflammation by IL-10 and regulatory T cells. PLoS One. 2012;7:e30883.

9. Liu J, Zhan W, Kim CJ, et al. IL-10-producing B cells are induced early in HIV-1 infection and suppress HIV-1-specific T cell responses. PLoS One. 2014;9:e89236.

10. Siewe B, Stapleton JT, Martinson J, et al. Regulatory B cell frequency correlates with markers of HIV disease progression and attenuates anti-HIV CD8+ T cell function in vitro. J Leukoc Biol. 2013;93:811-818.

11. Lin W, Cerny D, Chua E, et al. Human regulatory B cells combine phenotypic and genetic hallmarks with a distinct differentiation fate. J Immunol. 2014;193:2258-2266.

12. Lee KM, Stott RT, Zhao G, et al. TGF- $\beta$-producing regulatory B cells induce regulatory $\mathrm{T}$ cells and promote transplantation tolerance. Eur J Immunol. 2014;44:1728-1736.

13. Chen T, Song D, Min Z, et al. Perioperative dynamic alterations in peripheral regulatory $\mathrm{T}$ and $\mathrm{B}$ cells in patients with hepatocellular carcinoma. J Transl Med. 2012;10:14.

14. Olkhanud PB, Damdinsuren B, Bodogai M, et al. Tumor-evoked regulatory $\mathrm{B}$ cells promote breast cancer metastasis by converting resting CD4+ T cells to T-regulatory cells. Cancer Res. 2011; 71:3505-3515.

15. Carter NA, Vasconcellos R, Rosser EC, et al. Mice lacking endogenous IL-10-producing regulatory B cells develop exacerbated disease and present with an increased frequency of Th1/ Th17 but a decrease in regulatory $\mathrm{T}$ cells. J Immunol. 2011; 186:5569-5579.

16. Kessel A, Haj T, Peri R, et al. Human CD19(+)CD25(high) B regulatory cells suppress proliferation of $\mathrm{CD} 4(+) \mathrm{T}$ cells and enhance Foxp3 and CTLA-4 expression in T-regulatory cells. Autoimmun Rev. 2012;11:670-677.

17. Shetty S, Bruns T, Weston CJ, et al. Recruitment mechanisms of primary and malignant $\mathrm{B}$ cells to the human liver. Hepatology. 2012;56:1521-1531. 